\title{
Abatacept: a T-cell co-stimulation modulator for the treatment of rheumatoid arthritis
}

\author{
Andrew J K Östör
}

Received: 18 June 2008 / Accepted: 24 June 2008 / Published online: 1 August 2008

(C) Clinical Rheumatology 2008

\begin{abstract}
Rheumatoid arthritis (RA) is a debilitating autoimmune disease that has traditionally been treated with disease-modifying anti-rheumatic drugs (DMARDs). In the European Union (EU), patients who fail to respond to traditional DMARDs may receive tumor necrosis factor- $\alpha$ $(\mathrm{TNF}-\alpha)$ antagonists. However, approximately one-third of patients fail TNF- $\alpha$ antagonists due to adverse effects or lack of efficacy, and there are limited treatment options available to these patients. As knowledge of the underlying immunopathology of RA evolves, new strategies for inhibiting the inflammatory process have emerged. It is well known that activated $\mathrm{T}$ cells play a key role in orchestrating the immunopathological mechanisms of RA. Inhibiting the full activation of T cells is a rational strategy in the treatment of RA and represents a novel method of inhibiting disease activity, distinct from inflammatory cytokine blockade. Here, the safety and efficacy of abatacept, a selective T-cell co-stimulation modulator recently approved in the EU, is reviewed in patients with RA who have shown an inadequate response to TNF- $\alpha$ antagonists. In a randomized, placebocontrolled, double-blind, phase III trial of patients with an inadequate response to TNF- $\alpha$ antagonism, abatacept was effective in improving the signs and symptoms of RA, as well as patient-centered outcomes, such as fatigue, disability, and other mental and physical aspects of health-related quality of life. These improvements were sustained through 2 years during the open-label, long-term extension period. In this trial, abatacept demonstrated a safety and tolerability profile similar to placebo. Taken together, these data suggest that selective co-stimulation modulation with abatacept may
\end{abstract}

\section{A. J. K. Östör $(\square)$}

Rheumatology Research Unit, Box 194, Addenbrooke's Hospital, Cambridge University Hospitals NHS Foundation Trust,

Hills Road, Cambridge CB2 2QQ, UK

e-mail: andrew.ostor@addenbrookes.nhs.uk be a viable option for patients who are refractory to both traditional therapies and TNF- $\alpha$ antagonists.

Keywords Abatacept $\cdot$ Rheumatoid arthritis - Selective co-stimulation modulation $\cdot$ T cell $\cdot$ TNF- $\alpha$ antagonist

\section{Introduction}

For many adult patients with moderate or severe rheumatoid arthritis (RA) who are refractory to traditional non-biological disease-modifying anti-rheumatic drugs (DMARDs), the advent of the tumor necrosis factor (TNF)- $\alpha$ antagonists has provided much needed improvement in the clinical signs and symptoms of disease, as well as in patient-reported outcomes. However, experience from clinical practice has shown that not all of these difficult-to-treat patients benefit from TNF- $\alpha$ blockade; around one-third of patients either fail to respond to TNF- $\alpha$ antagonists [1] or discontinue therapy due to adverse effects [2]. As long as there are patients failing all current treatment options for RA, there is a need for new therapies, particularly ones with innovative mechanisms of action.

Abatacept (ORENCIA ${ }^{\circledR}$, Bristol-Myers Squibb, Princeton, NJ, USA) is the first in a new class of agents to be made available in Europe for use in patients with an inadequate response to TNF- $\alpha$ antagonists [3]. It is a soluble human fusion protein that selectively modulates T-cell activation without depleting $\mathrm{T}$ cells; it was approved in the European Union in May 2007 for the treatment of patients with moderate-to-severe RA who have had an inadequate response to, or intolerance of, other DMARDs, including at least one TNF- $\alpha$ antagonist.

This review provides an overview of RA and potential therapies before examining the rationale for modulating 
T-cell activation with abatacept. Evidence that supports the use of abatacept in TNF- $\alpha$ antagonist inadequate responders who have moderate-to-severe RA despite having had at least 3 months of treatment with a TNF$\alpha$ antagonist will then be discussed.

\section{Overview of RA and potential therapeutic options}

RA is a chronic, inflammatory disorder that affects between $0.5 \%$ and $1.0 \%$ of adult women and between $0.1 \%$ and $0.6 \%$ of adult men in the European Union [4]. It is a multisystem disease with signs and symptoms that include joint swelling, pain, prolonged morning joint stiffness, fatigue, muscle atrophy, and joint erosions $[5,6]$. The disease is usually polyarticular, with bilaterally symmetrical joint damage [7]. Extra-articular manifestations are common, including involvement of the eyes (sicca symptoms), lungs (pleural effusions, interstitial lung disease, nodules), skin (nodules, ulcers), heart (pericardial effusion, ischemic heart disease), and hematological system (anemia, thrombocytosis) [8-11]. The onset of symptoms predominantly occurs in patients over 50 years of age; however, there is a proclivity for women of child-bearing age [12]. The progressive pain and destruction of joints leads to increased disability and a significant impact on lifestyle, including an inability to work. RA is also associated with premature mortality, often from co-morbidities, such as cardiovascular and respiratory disease [11].

The traditional therapeutic pyramid for RA encouraged a cautious approach to treatment, initially involving nonsteroidal anti-inflammatory drugs (NSAIDs); low doses of DMARDs, such as methotrexate (MTX), sulfasalazine (SSZ), and hydroxychloroquine (HCQ); and corticosteroids, to attempt to reduce inflammation and slow the progression of disease [13]. However, the management of RA has changed markedly over the last decade. There has been a shift to more intensive treatment, including the early use of non-biologic DMARDs, prescribed alone or in combination with other non-biologic DMARDs. Latterly, if these therapies fail, biologic DMARDs have been used to attempt to improve outcome. Tumor necrosis factor- $\alpha$ antagonists are one such class of biologic DMARDs, including etanercept (ENBREL ${ }^{\circledR}$; Amgen, Thousand Oaks, CA, USA), infliximab (REMICADE ${ }^{\circledR}$; Centocor, Malvern, PA, USA) and adalimumab (HUMIRA ${ }^{\mathbb{}}$; Abbott Laboratories, Chicago, IL, USA). These agents have provided a valuable treatment option for patients with an inadequate response to, or an inability to tolerate, traditional non-biologic DMARDs.

Data have shown that patients who fail to respond to their first TNF- $\alpha$ antagonist may show an adequate response to a second TNF- $\alpha$ antagonist [2, 14-17]; however, evidence suggests that the probability of discontinuing therapy with a second TNF- $\alpha$ antagonist is higher than that of discontinuing the first [18]. Furthermore, retrospective clinical analysis has predicted that for patients who have failed two TNF- $\alpha$ antagonists, a third TNF- $\alpha$ antagonist is also likely to fail [1]. As there are currently no large, randomized clinical trials that have assessed switching between TNF- $\alpha$ antagonists, the benefits, or indeed limitations, of switching remain unclear.

Beyond cycling TNF- $\alpha$ antagonists, one option for this recalcitrant population would be to return to a non-biologic DMARD, such as MTX, SSZ, or HCQ, with or without the use of NSAIDs and corticosteroids; however, this approach is suboptimal for patients who have had an inadequate response to non-biologic DMARDs or for those who have specific co-morbidities.

Until recently, no treatment was available that had a mechanism of action (MoA) distinct from that of the TNF- $\alpha$ antagonists. However, there are now two available therapies, both of which target the underlying immunopathology of RA in a manner that differs from the TNF- $\alpha$ antagonists. Abatacept selectively modulates T-cell activation, whereas rituximab (MabThera ${ }^{\circledR}$, Hoffmann-La Roche) is a genetically engineered, chimeric, murine-human anti-CD20 monoclonal antibody, which depletes CD20-positive B cells by several proposed mechanisms [19-21]. Rituximab was approved in the EU in July 2006 for the treatment of patients with refractory RA, although it has been approved for some time for the treatment of non-Hodgkin's lymphoma. Both B-cell depletion with rituximab and T-cell modulation with abatacept provide therapeutic opportunities for patients with RA refractory to TNF- $\alpha$ blockade.

\section{The rationale for modulating T-cell activation in RA}

RA is an autoinflammatory disease involving multiple cell types, including dendritic cells, macrophages, monocytes, T and B cells, endothelial cells, and fibroblasts, which respond to genetic and environmental factors [22]. Although the etiology of RA remains obscure, it is thought that an unknown antigen triggers a pro-inflammatory signaling cascade within the synovium [22]. Activated $\mathrm{T}$ cells play a central role in this pathway by initiating this cascade with subsequent stimulation of macrophages and synovial fibroblasts to produce inflammatory cytokines, including TNF- $\alpha$, interleukin-2, and interferon- $\gamma$ [22]. T cells are also involved in activating $\mathrm{B}$ cells, which then produce autoantibodies, such as rheumatoid factor and anti-cyclic citrullinated peptides $[23,24]$. In addition, activated T cells produce the receptor activator of nuclear factor- $\mathrm{KB}$ (RANK) ligand that, in turn, binds to RANK on osteoclasts. This induces bone resorption and also stimulates chondrocytes to 
produce matrix metalloproteinases- 1 and -3 , leading to cartilage destruction [25]. By preventing full T-cell activation, many of these downstream events leading to joint destruction may be inhibited.

\section{Abatacept: a selective T-cell co-stimulation modulator}

By targeting T cells, abatacept has a fundamentally different MoA to other therapies for RA. However, rather than depleting $\mathrm{T}$ cells, abatacept modulates T-cell activation by binding to CD80 and CD86, thereby blocking interaction with CD28. For full activation, T cells require two distinct signals, the first occurring when major histocompatibility complex molecules on antigen-presenting cells (APCs) [26] present a peptide to the T-cell receptor. The second (co-stimulatory) signal is provided by further interaction between specific receptors on the APC and the T cell. If only one of the signals is transmitted, full activation of $\mathrm{T}$ cells does not occur; this is crucial to the MoA of abatacept.

Abatacept was developed to mimic the action of an endogenous protein, cytotoxic T-lymphocyte-associated antigen-4 (CTLA-4) [3], which also binds to CD80/CD86. CTLA-4 is a transmembrane protein expressed by activated $\mathrm{T}$ cells, which acts as a negative regulator of T-cell activation; it binds to CD80/CD86 with higher avidity than CD28, thereby inhibiting the positive co-stimulatory signal (Fig. 1a). As well as preventing this positive co-stimulation, the intracellular domain of endogenous CTLA-4 provides a negative co-stimulatory signal, suppressing further T-cell activation thus preventing the formation of subsequent components of the inflammatory cascade [3].
Fig. 1 Rationale for a T-cell co-stimulation modulator. a T-cell activation through the CD28:CD80/CD86 positive co-stimulatory pathway. In the normal immune response, CTLA-4 competes for CD28 binding to $\mathrm{CD} 80 / 86$ and produces a negative signal causing downregulation of T-cell activation. b Structure of abatacept, a T-cell co-stimulation modulator. Abatacept is a soluble recombinant fusion protein that consists of the extracellular domain of human CTLA-4 and a modified $\mathrm{Fc}$ domain from a human IgG1 protein [3]. APC Antigen-presenting cell, CTLA-4 cytotoxic T-lymphocyteassociated antigen-4, $\operatorname{Ig} G$ immunoglobulin G, $M H C$ major histocompatibility complex, $T C R$ T-cell receptor [Reproduced with permission from Barr C (2007) A Nursing Guide to Infusion Therapy with Abatacept for the Treatment of Rheumatoid Arthritis. Journal of Infusion Nursing 30(2):96-104]
A

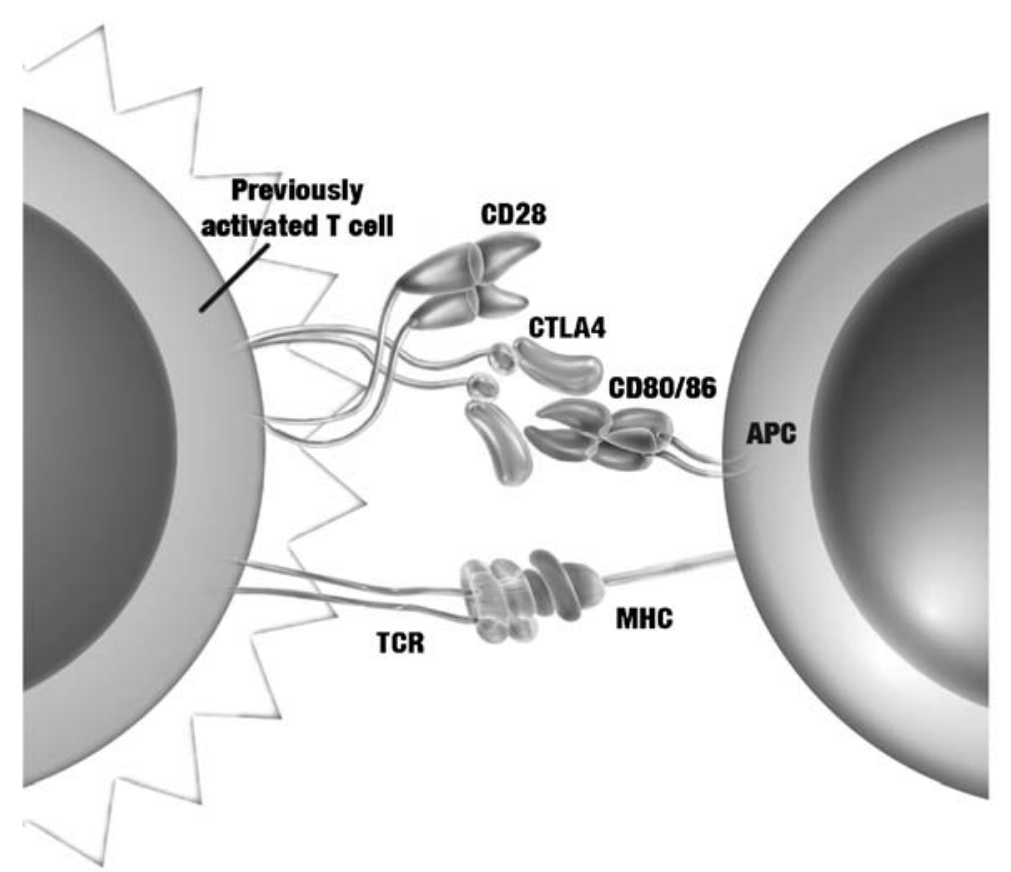

B

CTLA4

Abatacept

IgG1

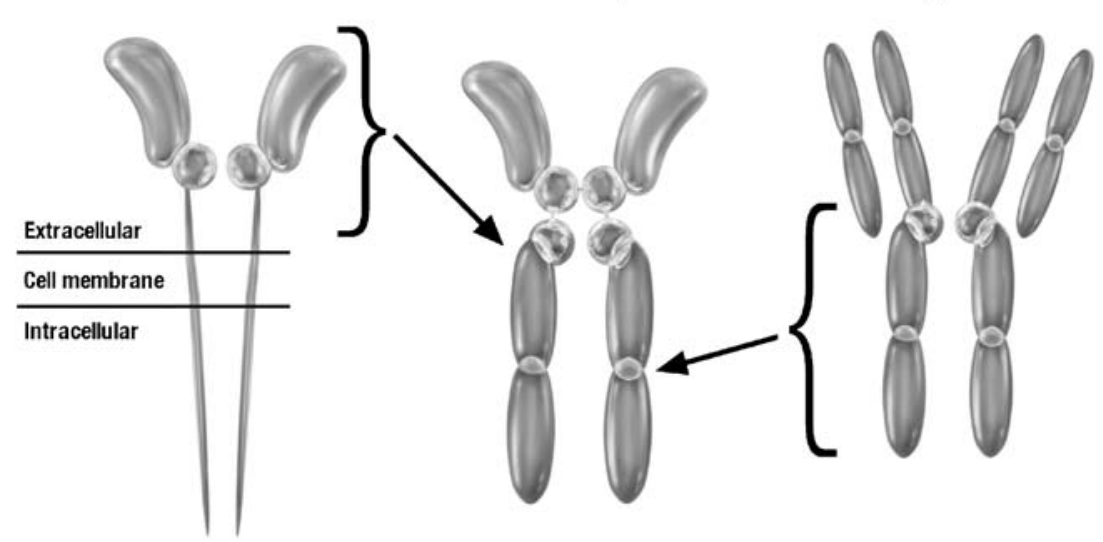


Abatacept is a fully soluble human fusion protein, incorporating only the extracellular domain of human CTLA-4, which is then linked to a modified Fc portion of human immunoglobulin G1 [3] (Fig. 1b). The modified Fc region helps stabilize the fusion protein, but is not therapeutically active; therefore, abatacept does not mediate complement-dependent cytotoxicity or antibody-dependent cell-mediated cytotoxicity (ADCC) [27]. These latter effects (complement-dependent cytotoxicity and ADCC) lead to cell lysis, which has been linked to adverse events (AEs), such as serious infusion reactions [27].

In relation to its unique MoA-selectively modulating T-cell co-stimulation-abatacept results in decreased proinflammatory cytokine secretion and decreased autoantibody production [28], thereby making T-cell co-stimulation modulation a rational strategy for RA therapy.

\section{Clinical overview of abatacept in patients with RA refractory to $\mathrm{TNF}-\alpha$ antagonists}

Abatacept has been shown to reduce disease activity and improve quality of life $(\mathrm{QoL})$ in this difficult-to-treat patient population [29, 30]. T-cell modulation with abatacept was evaluated in patients with moderate to severely active RA who had failed at least 3 months of TNF- $\alpha$ antagonist therapy in the ATTAIN (Abatacept Trial in Treatment of Anti-TNF INadequate responders) trial [29]. In this trial, 258 patients were randomized to abatacept and 133 patients to placebo, with both being administered by intravenous infusion. All patients in the trial received background DMARD therapy: of these $75.6 \%$ of patients randomized to the abatacept group and $82.0 \%$ of patients randomized to the placebo group were receiving background MTX. The patients who took part in the trial had long-standing, severe RA (mean disease duration \pm standard deviation $12.2 \pm 8.5$ years for the abatacept group and $11.4 \pm 8.9$ years for the placebo group). Both groups had a high number of tender and swollen joints at baseline with a mean of $31.2 \pm 13.0$ tender joints and $22.3 \pm$ 10.2 swollen joints in the abatacept group, and $32.8 \pm 13.4$ tender joints and $22.0 \pm 10.0$ swollen joints in the placebo group. Evaluation continued during a long-term extension (LTE) period, where all continuing patients received openlabel abatacept [30]. A total of 218 (84.5\%) patients originally randomized to abatacept and 99 (74.4\%) patients originally randomized to placebo entered the LTE, and 222 $(70.0 \%)$ of these completed a further 18 months of treatment.

\section{Efficacy with abatacept}

The American College of Rheumatology (ACR) response criteria were used to assess clinical efficacy [31]. The proportion of abatacept-treated patients who achieved an ACR 20 response progressively increased over the 6-month double-blind period and was significantly greater than the proportion of placebo-treated patients achieving an ACR 20 at all time points. The significant improvement seen with abatacept over placebo was observed as early as day 15 , after only one dose (Fig. 2a). At 1 month, over $40 \%$ of patients receiving abatacept had achieved an ACR 20 response compared with less than $20 \%$ of placebo-treated patients. Following 6 months of treatment, $50.4 \%$ of abatacept-treated patients had achieved an ACR 20 response, compared with $19.5 \%$ in the placebo group $(p<0.001$; Fig. $2 b)$. The difference between abatacept versus placebo in ACR 50 and 70 response rates was also significant after 6 months, with $20.3 \%$ and $10.2 \%$ of abatacept-treated patients attaining an ACR 50 or 70 response, respectively, versus $3.8 \%$ and $1.5 \%$ of the placebo-treated patients, respectively $(p<0.001$ and $p=0.003$ for ACR 50 and ACR 70, respectively; Fig. 2b).

The improvements in signs and symptoms observed at 6 months were maintained through 2 years in patients originally randomized to abatacept [30]. Using an intent-totreat (ITT) analysis that included patients who discontinued (defined as non-responders) at 2 years, ACR 20, 50, and 70 response rates were $56.2 \%, 33.2 \%$, and $16.1 \%$, respectively. Patients who were originally randomized to placebo and switched to abatacept at the beginning of the LTE period achieved improvements comparable to those of the patients originally in the abatacept group at 2 years (using nonresponder analysis, ACR 20, 50, and 70 responses for patients originally in the placebo group were $51.5 \%, 32.3 \%$, and $13.1 \%$, respectively). A post hoc analysis was also carried out on the ITT population using as-observed data; this analysis showed sustained improvements in ACR responses for the patients originally randomized to abatacept through 2 years (Fig. 2c). For ACR 20 and 50, the 95\% confidence intervals (CIs) do no overlap at 6 months and 2 years, suggesting that there was an improved response over time. The post hoc analysis may be more relevant over the long term as it follows only those patients who continue therapy in the study.

Disease activity was also assessed in this trial using the Disease Activity Score 28 (DAS28). At baseline, these patients had severe disease, with a DAS28 score of $6.5 \pm 0.9$ in the abatacept group and $6.5 \pm 0.8$ in the placebo group [29]. Following 6 months of treatment, $17.1 \%$ of patients in the abatacept group had low disease activity (LDAS; defined as a DAS28 score of $\leq 3.2$ ) after 6 months compared with $3.1 \%$ of patients in the placebo group $(p<$ 0.001; Fig. 3a) [29]. In addition, $10 \%$ of abatacept-treated patients were in disease remission (defined by a DAS28 [CRP] <2.6), compared with $0.8 \%$ of placebo-treated patients $(p<0.001$; Fig. 3a). At 2 years, using the post hoc as-observed analyses, nearly one-fifth of patients originally randomized to receive abatacept achieved DAS28 
Fig. 2 ATTAIN phase III trial: American College of Rheumatology response rates during the double-blind and long-term extension periods [29, 30]. a ACR 20 response rate at each post-baseline visit during the 6-month trial (double-blind period). The numbers below the panel are the numbers of patients with a response who were assessed. b ACR 20, ACR 50 , and ACR 70 responses at 6 months (double-blind period). Two patients in the abatacept group were excluded from the efficacy analysis at 6 months because of a protocol violation. c ACR 20, ACR 50, and ACR 70 response rates using a post hoc as-observed analysis of patients originally randomized to abatacept at the beginning of the 6-month double-blind period and continued receiving abatacept during the 18-month LTE. $A C R$ American College of Rheumatology, LTE long-term extension [Genovese MC, Becker JC, Schiff M et al. (2005). Abatacept for rheumatoid arthritis refractory to tumor necrosis factor alpha inhibition. N Engl J Med 353:1114-1123. Copyright (C) 2005 Massachusetts Medical Society. All rights reserved; Genovese MC, Schiff M, Luggen $M$ et al. (2007) Efficacy and safety of the selective co-stimulation modulator abatacept following 2 years of treatment in patients with rheumatoid arthritis and an inadequate response to anti-TNF therapy. Ann Rheum Dis 67:547-554. Reproduced with permission from the BMJ Publishing Group]
A

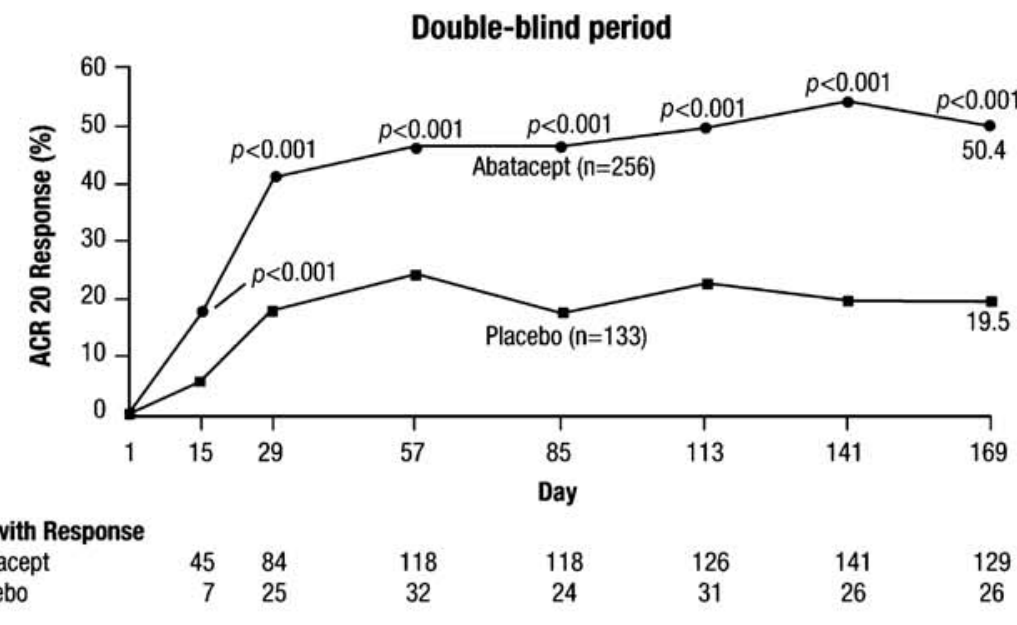

B

Double-blind period

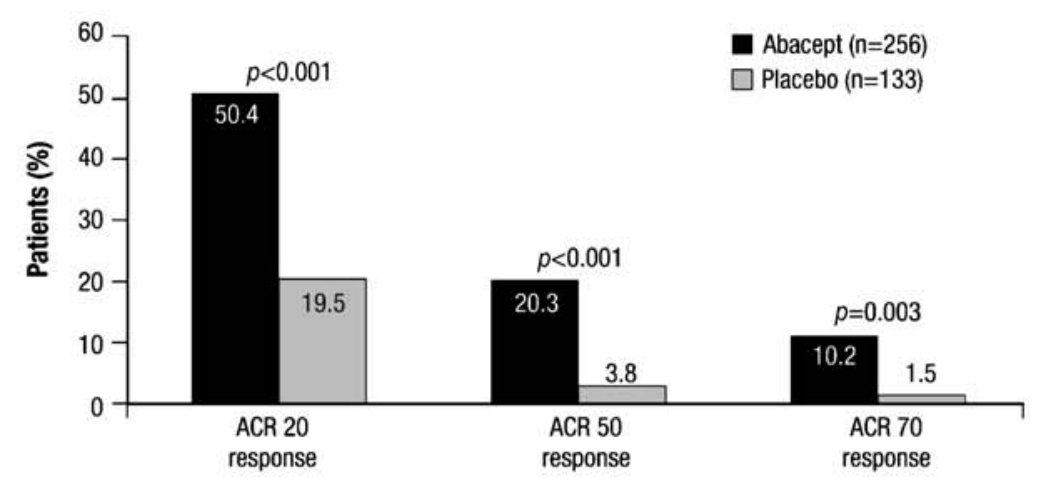

C

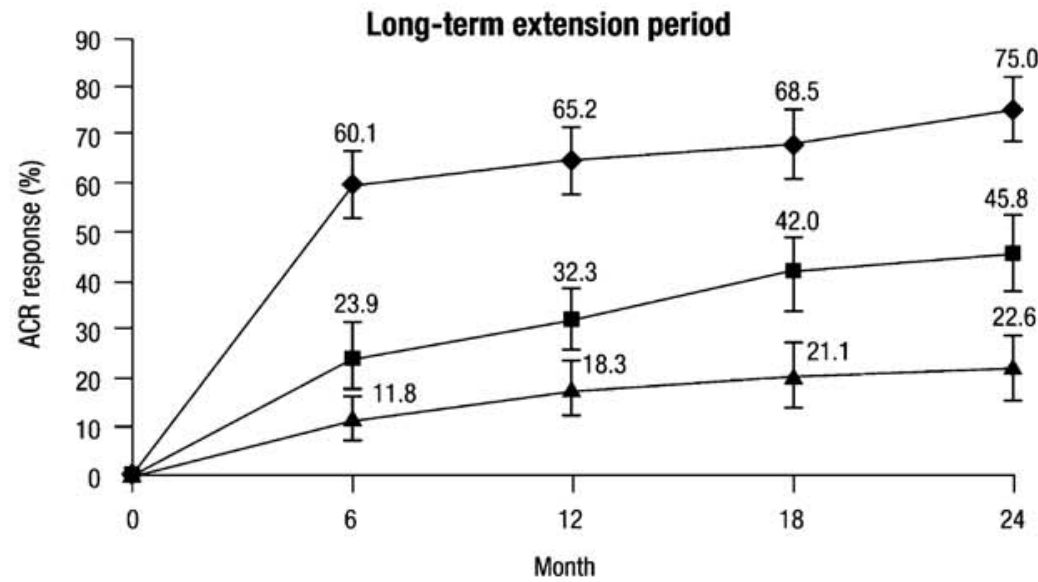

\begin{tabular}{|l|c|c|c|c|}
\hline \multicolumn{5}{|c|}{ N-numbers for the post-hoc as-observed analysis (figure 2C) } \\
\hline Assessment & $\begin{array}{c}\text { Month 6 } \\
\text { (n) }\end{array}$ & $\begin{array}{c}\text { Month 12 } \\
\text { (n) }\end{array}$ & $\begin{array}{c}\text { Month 18 } \\
\text { (n) }\end{array}$ & $\begin{array}{c}\text { Month 24 } \\
\text { (n) }\end{array}$ \\
\hline ACR 20 & 208 & 198 & 168 & 156 \\
\hline ACR 50 & 209 & 201 & 169 & 153 \\
\hline ACR 70 & 212 & 202 & 171 & 155 \\
\hline
\end{tabular}

$\multimap$ ACR $20 \rightarrow-A C R 50 \rightarrow$ ACR 70 
(CRP)-defined remission, showing that the proportion of patients in remission was maintained through the 2 years (Fig. 3b) [30]. The proportion of patients who achieved LDAS increased from $18.3 \%(95 \% \mathrm{CI} ; 13.0,23.5)$ at the end of the double-blind period to $32.0 \%(24.6,39.4)$ at 2 years with no overlap in the $95 \%$ CIs between 6 months and 2 years (Fig. 3b).

\section{Improvements in physical function and health-related quality of life with abatacept}

T-cell co-stimulation modulation has been shown to provide clinically meaningful and statistically significant improvements in multiple aspects of QoL, including mental and physical function [5, 29]. Improvements in measures such as fatigue and sleep quality have also been observed in

A
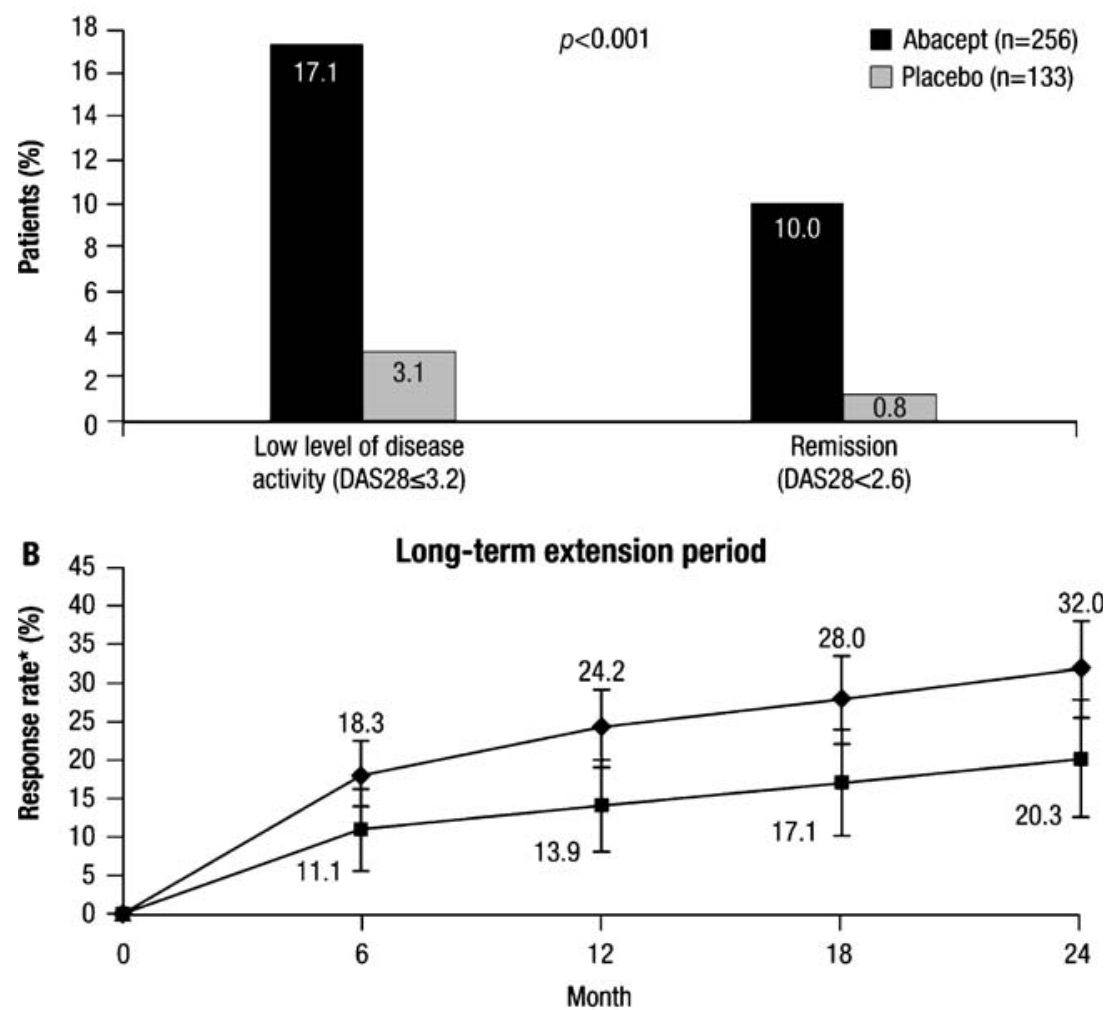

\begin{tabular}{|l|c|c|c|c|}
\hline Assessment & $\begin{array}{c}\text { Month 6 } \\
(\mathrm{n})\end{array}$ & $\begin{array}{c}\text { Month 12 } \\
(\mathrm{n})\end{array}$ & $\begin{array}{c}\text { Month 18 } \\
(\mathrm{n})\end{array}$ & $\begin{array}{c}\text { Month 24 } \\
(\mathrm{n})\end{array}$ \\
\hline LDAS & 208 & 194 & 164 & 153 \\
\hline $\begin{array}{l}\text { DAS28 (CRP)- } \\
\text { defined remission }\end{array}$ & 208 & 194 & 164 & 153 \\
\hline
\end{tabular}

Fig. 3 ATTAIN phase III trial: the proportion of patients experiencing disease remission and low disease activity during the double-blind and long-term extension periods [29, 30]. a DAS28 (CRP)-defined remission (DAS $[\mathrm{CRP}]<2.6$ ) and LDAS (DAS [CRP] $\leq 3.2$ ) at 6 months (double-blind period). Two patients in the abatacept group were excluded from the efficacy analysis at 6 months because of a protocol violation. b DAS28 (CRP)-defined remission and LDAS through 2 years using post hoc as-observed analysis of patients originally randomized to abatacept at the beginning of the 6-month double-blind period and continued receiving abatacept during the 18month LTE period. $L D A S$ Low disease activity score, DAS disease activity score, CRP C-reactive protein, LTE long-term extension [Genovese MC, Becker JC, Schiff M et al. (2005). Abatacept for rheumatoid arthritis refractory to tumor necrosis factor alpha inhibition. N Engl J Med 353:1114-1123. Copyright (C) 2005 Massachusetts Medical Society. All rights reserved; Genovese MC, Schiff M, Luggen $\mathrm{M}$ et al. (2007) Efficacy and safety of the selective co-stimulation modulator abatacept following 2 years of treatment in patients with rheumatoid arthritis and an inadequate response to anti-TNF therapy. Ann Rheum Dis 67:547-554. Reproduced with permission from the BMJ Publishing Group] 
A

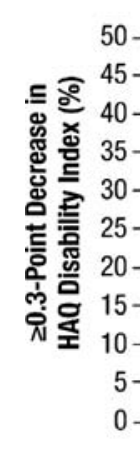

A
Double-blind period

\section{B}

\section{Long-term extension period}

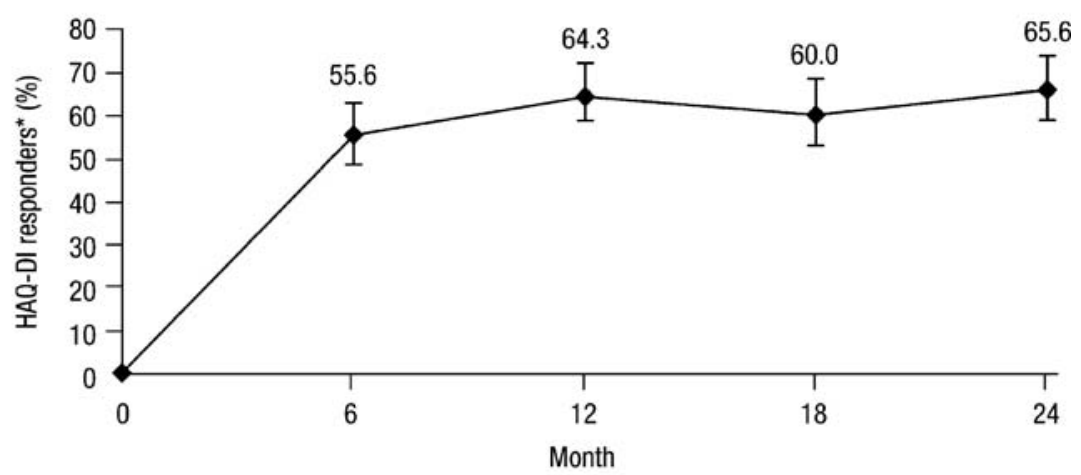

\begin{tabular}{|l|c|c|c|c|}
\hline Assessment & $\begin{array}{c}\text { Month 6 } \\
\text { (n) }\end{array}$ & $\begin{array}{c}\text { Month 12 } \\
\text { (n) }\end{array}$ & $\begin{array}{c}\text { Month 18 } \\
\text { (n) }\end{array}$ & $\begin{array}{c}\text { Month 24 } \\
\text { (n) }\end{array}$ \\
\hline HAQ-DI & 207 & 199 & 165 & 154 \\
\hline
\end{tabular}

$\multimap$ Abatacept $10 \mathrm{mg} / \mathrm{kg}$ plus DMARDs

Fig. 4 ATTAIN phase III trial: physical function during the doubleblind and long-term extension periods [29, 30]. a The proportion of patients achieving clinically meaningful HAQ-DI responses at 6 months (double-blind period. b The proportion of patients achieving clinically meaningful HAQ-DI responses through 2 years using post hoc as-observed analysis of patients originally randomized to abatacept at the beginning of the 6-month double-blind period and continued receiving abatacept during the 18-month LTE period. A clinically meaningful improvement from baseline was considered to be $\geq 0.3$ units in the HAQ-DI. Bars on the 2-year data represent $95 \%$ CI. *HAQ-DI responses are based on the ITT population for patients originally randomized to receive abatacept, with data available at the

patients receiving abatacept $[29,32]$, thereby enhancing the patient's ability to live a normal life.

Improvements in physical function were assessed in the ATTAIN trial using the Health-Assessment Questionnaire Disability Index (HAQ-DI). At baseline, patients exhibited substantial impairment in physical function, with an average HAQ-DI score of 1.8 across the treatment groups. In this trial, a patient was considered a HAQ-DI responder if they achieved an improvement of at least 0.3 units from baseline (exceeding the 0.22 value that is considered clinically visit of interest (post hoc as-observed analysis). HAQ-DI Health Assessment Questionnaire Disability Index, ITT intent to treat, LTE long-term extension [Genovese MC, Becker JC, Schiff M et al. (2005). Abatacept for rheumatoid arthritis refractory to tumor necrosis factor alpha inhibition. N Engl J Med 353:1114-1123. Copyright (C) 2005 Massachusetts Medical Society. All rights reserved; Genovese MC, Schiff M, Luggen M et al. (2007) Efficacy and safety of the selective co-stimulation modulator abatacept following 2 years of treatment in patients with rheumatoid arthritis and an inadequate response to antiTNF therapy. Ann Rheum Dis 67:547-554. Reproduced with permission from the BMJ Publishing Group]

meaningful [33]). After 6 months of treatment, $47.3 \%$ of patients receiving abatacept had a clinically meaningful improvement in their HAQ-DI score, whereas significantly fewer placebo-treated patients $(23.3 \%)$ had a similar improvement ( $p<0.001$; Fig. 4a) [29]. The abatacept group showed a significant improvement from baseline of 0.45 units in their HAQ-DI score after 6 months, compared with an improvement of 0.11 units shown by the placebo group $(p<0.001)$ [29]. The clinically meaningful improvements seen at 6 months were maintained through 2 years 


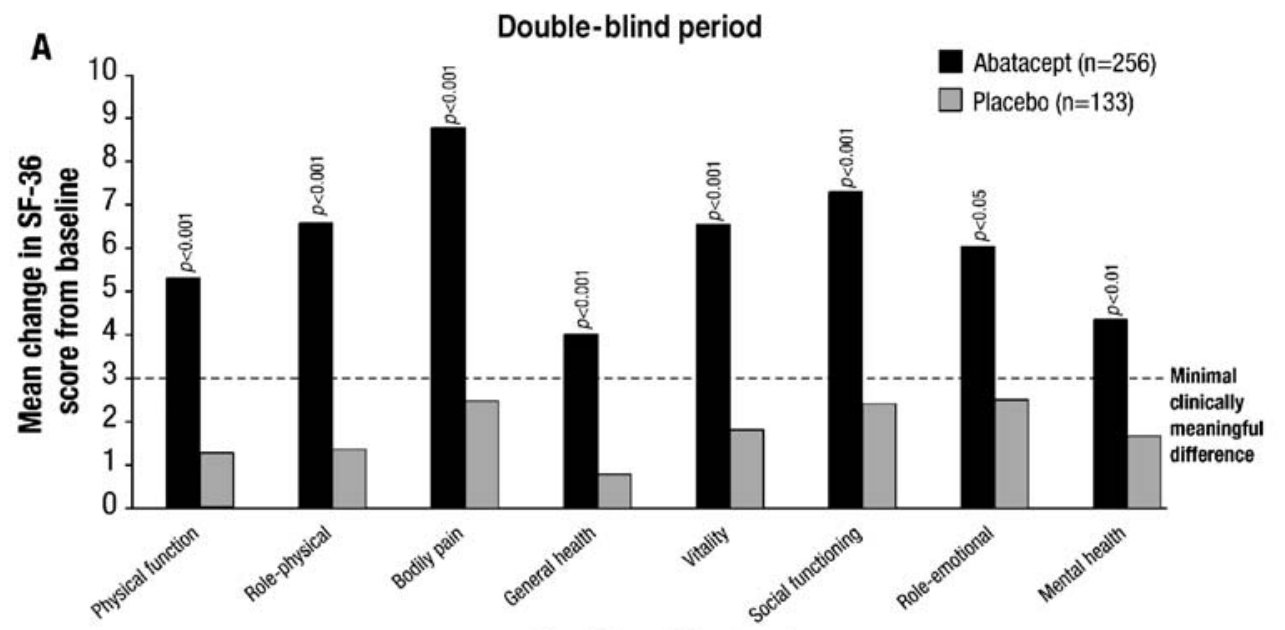

Short Form-36 subscale
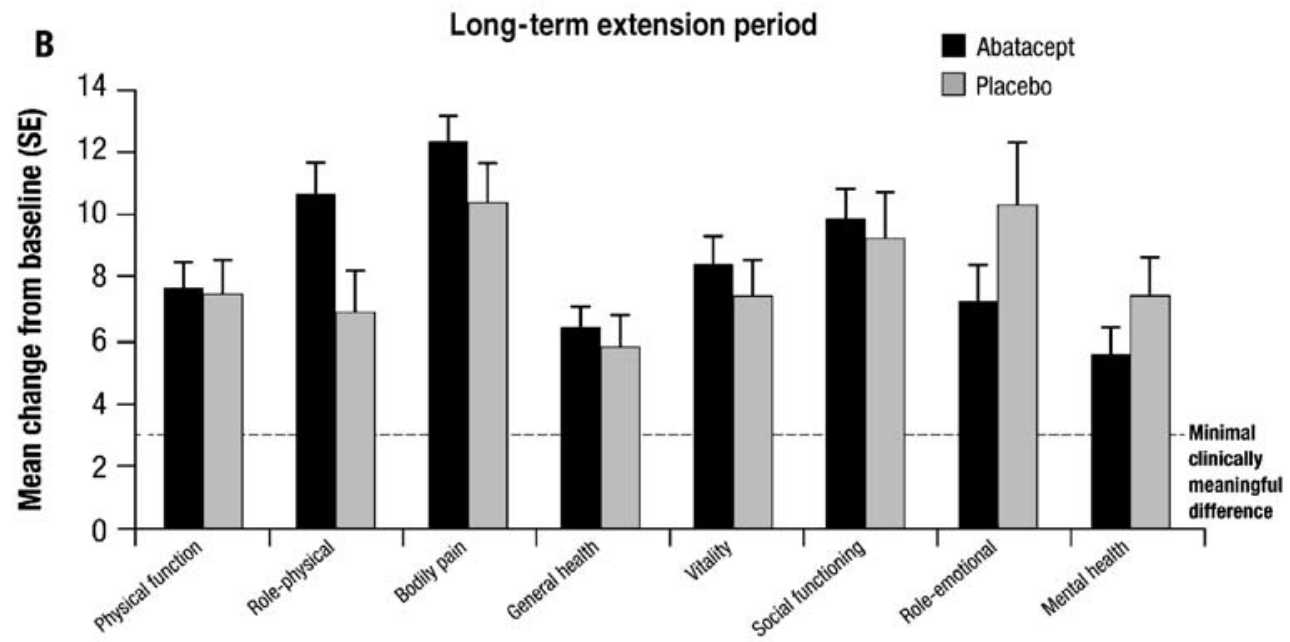

Short Form-36 subscale

C

Abatacept

$\square$ Placebo

$\otimes$ Placebo to abatacept

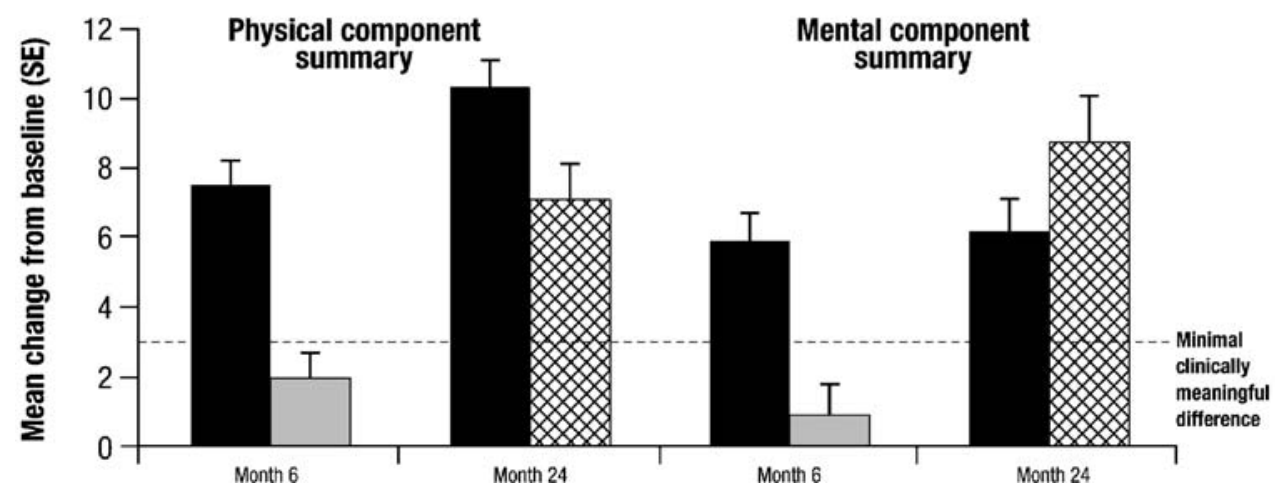

(Fig. 4b); approximately two-thirds of patients originally randomized to receive abatacept experienced an improvement in physical function at 2 years using the post hoc as-observed analyses.

Although improvement in physical function is salient, other aspects of patient health, including mental state and overall QoL are becoming increasingly important when measuring the efficacy of a therapy for RA [5, 33, 34]. Improvements in health-related QoL were evaluated in the ATTAIN trial using the Medical Outcomes Study Short Form-36 (SF-36). The SF-36 consists of 36 items, 35 of which evaluate eight dimensions of health, including four 
4 Fig. 5 ATTAIN phase III trial: health-related quality of life during the double-blind and long-term extension period [29, 30]. a The effect of abatacept and placebo on the mean change from baseline in the individual subscales of the SF-36 at 6 months (double-blind period). For the double-blind period, two patients in the abatacept group were excluded from the efficacy analysis because of a protocol violation. b The effect of abatacept on the mean change from baseline in the individual subscales of the SF-36 at 2 years using post hoc as-observed analysis of patients originally randomized to abatacept at the beginning of the 6-month double-blind period and continued receiving abatacept during the 18-month LTE period [35]. Scores can range from 0 to 100, with higher scores indicating a better quality of life. An improvement of 3 units is considered clinically meaningful [36], as indicated by the dotted line. $\mathbf{c}$ Mean change from baseline in the PCS and MCS scores at 6 months and 2 years in all patients who entered the LTE period. Patients who received placebo plus DMARDs during the initial doubleblind period were reallocated to abatacept plus DMARDs at the start of the LTE. Both double-blind (day 1 to month 6) and LTE (month 6 to month 24) data are represented. SF-36 Short form-36, LTE long-term extension, $P C S$ physical component summary, $M C S$ mental component summary, DMARD disease-modifying anti-rheumatic drug [Genovese MC, Becker JC, Schiff M et al. (2005). Abatacept for rheumatoid arthritis refractory to tumor necrosis factor alpha inhibition. $\mathrm{N}$ Engl $\mathrm{J}$ Med 353:1114-1123. Copyright (C) 2005 Massachusetts Medical Society. All rights reserved; Genovese MC, Schiff M, Luggen M et al. (2007) Efficacy and safety of the selective co-stimulation modulator abatacept following 2 years of treatment in patients with rheumatoid arthritis and an inadequate response to anti-TNF therapy. Ann Rheum Dis 67:547-554. Reproduced with permission from the BMJ Publishing Group]

physical (physical functioning, role-physical, bodily pain, general health) and four mental subscales (vitality, social functioning, role-emotional, mental health), as well as overall summary scores for the physical and mental components. During the double-blind period, patients receiving abatacept showed greater improvements than placebo-treated patients in the change from baseline in the scores for all eight physical and mental subscales of the SF-36 and both component summary scores (Fig. 5a) [29] and these were maintained or increased through 2 years (Fig. 5b) [30]. For the mental component summary of the SF-36 analyses, the mean change from baseline ( \pm standard deviation) was $5.4 \pm$ 11.7 units after 6 months of abatacept therapy compared with $1.7 \pm 0.2$ units for the placebo group $(p=0.0025)$ [5]. At 2 years, the greatest improvements were observed in the physical component subscales including bodily pain and role-physical [30]. The mean improvements from baseline in the physical and mental component summaries (PCS and MCS, respectively) at 6 months and 2 years for patients originally randomized to receive abatacept were 38.1 and 48.3 , respectively, which are 10.3 and 6.2 point improvements from baseline (Fig. 5c).

Patients were also asked to monitor the degree of fatigue they experienced using a visual analogue scale (VAS) ranging from no fatigue at score ' 0 ' to extreme fatigue at score ' 100 '. The abatacept-treated group improved significantly more than the placebo-treated group at 6 months, with the mean fatigue VAS score decreasing by $22.1 \pm 28.6$ in the abatacept group compared with a decrease of $5.3 \pm 27.4$ for the placebo group $(p<0.0001)$ [5]. This significant and clinically meaningful improvement in physical and mental health, including fatigue, is important, particularly in this population of patients who have severe disease and limited treatment options.

\section{Safety of abatacept in the ATTAIN trial}

During the double-blind period, the overall frequencies of AEs and serious AEs (SAEs) were not significantly different in abatacept- and placebo-treated patients. A total of $79.5 \%$ of patients receiving abatacept and $71.4 \%$ of patients receiving placebo reported AEs during the doubleblind period $(p=0.08)$; SAEs were also reported with similar frequency in the abatacept $(10.5 \%)$ and placebo $(11.3 \%)$ groups $(p=0.81)$ [29]. Similar types of AEs, including infusion-related events, were also reported during the LTE period. Incidence rates of SAEs were consistent for the double-blind and cumulative (double-blind plus LTE) periods, with rates of 25.6 and 23.4/100 patient-years, respectively. The most frequent SAEs for the cumulative period were reported to be infestations and infections, and malignancies. A total of 11 malignancies were reported during the cumulative period, including non-melanomatous skin cancers, basal cell carcinoma ( $n=3$ patients) and squamous cell carcinoma ( $n=2$ patients). During the double-blind period, the overall frequency of discontinuation due to AEs or SAEs was low and was similar in both groups (discontinuations due to AEs for the abatacept group versus placebo group: nine patients [3.5\%] versus five patients [3.8\%], respectively; discontinuations due to SAEs for abatacept group versus placebo group: seven patients [2.7\%] versus two patients [1.5\%] placebo group, respectively; $p=0.89$ ). During the cumulative period, a total of 25 patients discontinued due to AEs and 18 patients discontinued due to SAEs.

There was no significant difference in the frequency of infections between the abatacept and placebo groups during the double-blind period (37.6\% versus $32.3 \%$, respectively; $p=0.30$ ). Generally, infections were reported as mild to moderate in intensity, with nasopharyngitis, sinusitis, upper respiratory tract infections, and bronchitis being the most frequent, and no unusual or opportunistic infections were observed. For the double-blind period, discontinuations due to infection were $0.8 \%$ in the abatacept group compared with $1.5 \%$ in the placebo group $(p=0.61)$. During the cumulative period, overall infections and serious infections occurred in 234 and 25 patients, with incidence rates of 89.4 and 5.0/100 patients-years, respectively. These rates were similar to those reported for the double-blind period 
(108.8 and 5.3/100 patients-years, respectively). For the cumulative period, discontinuations due to infections occurred in five patients, three of which had serious infections (pneumonia, streptococcal sepsis, and subacute endocarditis).

Overall, the 30-min intravenous abatacept infusion was well tolerated by patients in this trial [29] and did not require premedication [37]. The incidence of infusion reactions was low, reported by only $5 \%$ of abatacept-treated patients compared with $3 \%$ of the placebo-treated patients ( $p=$ 0.35 ), and no severe or very severe reactions were observed in either group during the double-blind period. The most common infusion events experienced were dizziness (reported by $1.6 \%$ of the abatacept-treated patients and $0.0 \%$ of placebo-treated patients; $p=0.30$ ) and headache (reported by $1.2 \%$ of abatacept-treated patients and $0.8 \%$ of placebo-treated patients; $p=1.0$ ). The incidence of infusion events during the cumulative period was similar to those reported during the double-blind period.

In other randomized clinical trials, including the AIM (Abatacept in Inadequate responders to Methotrexate) trial, the use of abatacept earlier in the treatment paradigm in patients with an inadequate response to MTX has been evaluated [37, 38]. Abatacept demonstrated favorable efficacy in this trial, including inhibition of radiographic progression through 2 years [39], which was not measured in the ATTAIN trial, with an acceptable safety profile. Abatacept is not indicated for use in this patient population in the EU, but it has been approved in other countries, including the USA, for the treatment of patients with moderate to severe RA who have shown an inadequate response to non-biologic DMARDs.

\section{Conclusions}

The ultimate goal of treating autoimmune diseases, such as RA, should be to suppress the aberrant immune response in a specific and long-term manner without compromising normal immunity. Although many patients with RA are successfully treated with traditional, non-biologic DMARDs, until recently there has been a dearth of treatment options available to patients who have failed these agents. The advent of TNF- $\alpha$ antagonists has provided hope to these patients; however, some patients also fail to respond to these therapies, lose their response over time, or suffer intolerable side effects $[2,14,18]$. Hence, there is a need for treatments with distinct MoAs for use in this refractory population.

Selective T-cell co-stimulation modulation with abatacept offers a potentially valuable therapeutic option for patients with RA who are refractory to TNF- $\alpha$ antagonist therapy and have progressed through the treatment paradigm and are more likely to have severe disease.
Abatacept has been shown to be efficacious in patients with moderate to severely active RA who had failed at least 3 months of TNF- $\alpha$ antagonist therapy in the ATTAIN trial. The data presented here from the ATTAIN trial demonstrates that abatacept provides significant improvements in the signs and symptoms of RA, as well as improves QoL outcomes, including fatigue, mental health, and physical disability for up to 2 years $[5,29,30,32]$. Data from this trial also demonstrate that abatacept has an acceptable safety and tolerability profile with a low (and not dissimilar to placebo) frequency of infusion reactions and serious infections.

Although longer-term data on efficacy and safety are needed to further validate T-cell co-stimulation modulation as a therapeutic approach for RA, abatacept clearly contributes to bridging the unmet need for managing disease progression in patients for whom TNF- $\alpha$ antagonist therapy has failed.

Acknowledgments The author would like to thank Celia Parkyn, Ph.D., Medicus International, for her editorial support. Editorial support was funded by Bristol-Myers Squibb, Uxbridge, Middlesex, United Kingdom.

Conflict of interest statement Andrew Östör has received funding for various activities from multiple pharmaceutical companies, including Schering-Plough, Abbott, Wyeth, Roche, BMS, and MSD.

\section{References}

1. Solau-Gervais E, Laxenaire N, Cortet B et al (2006) Lack of efficacy of a third tumour necrosis factor alpha antagonist after failure of a soluble receptor and a monoclonal antibody. Rheumatology (Oxford) 45:1121-1124

2. Iannone F, Trotta F, Montecucco C et al (2007) Etanercept maintains the clinical benefit achieved by infliximab in patients with rheumatoid arthritis who discontinued infliximab because of side effects. Ann Rheum Dis 66:249-252

3. Linsley PS, Brady W, Urnes M et al (1991) CTLA-4 is a second receptor for the B cell activation antigen B7. J Exp Med 174:561569

4. Symmons DP (2002) Epidemiology of rheumatoid arthritis: determinants of onset, persistence and outcome. Best Pract Res Clin Rheumatol 16:707-722

5. Westhovens R, Cole JC, Li T et al (2006) Improved health-related quality of life for rheumatoid arthritis patients treated with abatacept who have inadequate response to anti-TNF therapy in a double-blind, placebo-controlled, multicentre randomized clinical trial. Rheumatology (Oxford) 45:1238-1246

6. Arnett FC, Edworthy SM, Bloch DA et al (1988) The American Rheumatism Association 1987 revised criteria for the classification of rheumatoid arthritis. Arthritis Rheum 31:315-324

7. Clarke GS, Buckland-Wright JC, Grahame R (1994) Symmetry of radiological features in the wrist and hands of patients with early to moderate rheumatoid arthritis: a quantitative microfocal radiographic study. Br J Rheumatol 33:249-254

8. Piper H, Douglas KM, Treharne GJ et al (2007) Prevalence and predictors of ocular manifestations of RA: is there a need for routine screening? Musculoskeletal Care 5:102-117 
9. Kim DS (2006) Interstitial lung disease in rheumatoid arthritis: recent advances. Curr Opin Pulm Med 12:346-353

10. Levin J, Werth VP (2006) Skin disorders with arthritis. Best Pract Res Clin Rheumatol 20:809-826

11. Voskuyl AE (2006) The heart and cardiovascular manifestations in rheumatoid arthritis. Rheumatology (Oxford) 45:IV4-IV7

12. Cooper G, Stroehla B (2003) The epidemiology of autoimmune diseases. Autoimmun Rev 2:119-125

13. Smolen JS, Aletaha D, Koeller M et al (2007) New therapies for treatment of rheumatoid arthritis. Lancet 370:1861-1874

14. Hyrich KL, Lunt M, Watson KD et al (2007) Outcomes after switching from one anti-tumor necrosis factor alpha agent to a second anti-tumor necrosis factor alpha agent in patients with rheumatoid arthritis: results from a large UK national cohort study. Arthritis Rheum 56:13-20

15. Di Poi E, Perin A, Morassi MP et al (2007) Switching to etanercept in patients with rheumatoid arthritis with no response to infliximab. Clin Exp Rheumatol 25:85-87

16. Cohen G, Courvoisier N, Cohen JD et al (2005) The efficiency of switching from infliximab to etanercept and vice-versa in patients with rheumatoid arthritis. Clin Exp Rheumatol 23:795-800

17. Wick MC, Ernestam S, Lindblad S et al (2005) Adalimumab (Humira) restores clinical response in patients with secondary loss of efficacy from infliximab (Remicade) or etanercept (Enbrel): results from the STURE registry at Karolinska University Hospital. Scand J Rheumatol 34:353-358

18. Gomez-Reino JJ, Carmona L (2006) Switching TNF antagonists in patients with chronic arthritis: an observational study of 488 patients over a four-year period. Arthritis Res Ther 8:R29

19. Browning JL (2006) B cells move to centre stage: novel opportunities for autoimmune disease treatment. Nat Rev Drug Discov 5:564-576

20. Reff ME, Carner K, Chambers KS et al (1994) Depletion of B cells in vivo by a chimeric mouse human monoclonal antibody to CD20. Blood 83:435-445

21. Maloney DG, Smith B, Rose A (2002) Rituximab: mechanism of action and resistance. Semin Oncol 29:2-9

22. Choy EH, Panayi GS (2001) Cytokine pathways and joint inflammation in rheumatoid arthritis. N Engl J Med 344:907916

23. Yamada A, Salama AD, Sayegh MH (2002) The role of novel T cell costimulatory pathways in autoimmunity and transplantation. J Am Soc Nephrol 13:559-575

24. Burkhardt H, Sehnert B, Bockermann R et al (2005) Humoral immune response to citrullinated collagen type II determinants in early rheumatoid arthritis. Eur J Immunol 35:1643-1652

25. Tanaka S, Nakamura K, Takahasi N et al (2005) Role of RANKL in physiological and pathological bone resorption and therapeutics targeting the RANKL-RANK signaling system. Immunol Rev 208:30-49
26. Cravens PD, Lipsky PE (2002) Dendritic cells, chemokine receptors and autoimmune inflammatory diseases. Immunol Cell Biol 80:497-505

27. Davis PM, Abraham R, Xu L et al (2007) Abatacept binds to the Fc receptor CD64 but does not mediate complement-dependent cytotoxicity or antibody-dependent cellular cytotoxicity. J Rheumatol 34:2204-2210

28. Weisman MH, Durez P, Hallegua D et al (2006) Reduction of inflammatory biomarker response by abatacept in treatment of rheumatoid arthritis. J Rheumatol 33:2162-2166

29. Genovese MC, Becker JC, Schiff M et al (2005) Abatacept for rheumatoid arthritis refractory to tumor necrosis factor alpha inhibition. N Engl J Med 353:1114-1123

30. Genovese MC, Schiff M, Luggen M et al (2007) Efficacy and safety of the selective co-stimulation modulator abatacept following 2 years of treatment in patients with rheumatoid arthritis and an inadequate response to anti-TNF therapy. Ann Rheum Dis 67: $547-554$

31. Felson DT, Anderson JJ, Boers M et al (1995) American College of Rheumatology. Preliminary definition of improvement in rheumatoid arthritis. Arthritis Rheum 38:727-735

32. Wells G, Li T, Maxwell L et al (2007) Determining the minimal clinically important differences in activity, fatigue, and sleep quality in patients with rheumatoid arthritis. J Rheumatol 34:280 289

33. Wells GA, Tugwell P, Kraag GR et al (1993) Minimum important difference between patients with rheumatoid arthritis: the patient's perspective. J Rheumatol 20:557-560

34. Kirwan JR, Minnock P, Adebajo A et al (2007) Patient perspective: fatigue as a recommended patient centered outcome measure in rheumatoid arthritis. J Rheumatol 34:1174-1177

35. Ware JE Jr., Sherbourne CD (1992) The MOS 36-item short-form health survey (SF-36). I. Conceptual framework and item selection. Med Care 30:473-483

36. Samsa G, Edelman D, Rothman ML et al (1999) Determining clinically important differences in health status measures: a general approach with illustration to the Health Utilities Index Mark II. Pharmacoeconomics 15:141-155

37. Kremer JM, Genant HK, Moreland LW et al (2006) Effects of abatacept in patients with methotrexate-resistant active rheumatoid arthritis: a randomized trial. Ann Intern Med 144:865876

38. Kremer JM, Genant HK, Moreland LW et al (2008) Results of a two-year followup study of patients with rheumatoid arthritis who received a combination of abatacept and methotrexate. Arthritis Rheum 58:953-963

39. Genant HK, Peterfy CG, Westhovens R et al (2008) Abatacept inhibits structural damage progression in rheumatoid arthritis: results from the long-term extension of the AIM trial. Ann Rheum Dis 67:1084-1089 